doi:10.13108/2020-12-3-30

\title{
INVARIANT SUBSPACES IN HALF-PLANE
}

\author{
A.S. KRIVOSHEEV, O.A. KRIVOSHEEVA
}

\begin{abstract}
We study subspaces of functions analytic in a half-plane and invariant with respect to the differentiation operator. A particular case of an invariant subspace is a space of solutions a linear homogeneous differential equation with constant coefficients. It is known that each solution of such equations is a linear combination of primitive solutions, which are exponential monomials with exponents being possibly multiple zeroes of a characteristic polynomial. The existence of such representation is called Euler fundamental principle. Other particular cases of invariant subspaces are spaces of solutions of linear homogeneous differential, difference and differential-difference equations with constant coefficients of both finite and infinite orders as well as of more general convolution equations and the systems of them. In the work we study the issue on fundamental principle for arbitrary invariant subspaces for arbitrary invariant subspaces of analytic functions in a half-plane. In other words, we study representation of all functions in an invariant subspace by the series of exponential monomials. These exponential monomials are eigenfunctions and adjoint functions for the differentiation operator in an invariant subspace. In the work we obtain a decomposition of an arbitrary invariant subspace of analytic functions into a sum of two invariant subspaces. We prove that the invariant subspace in an unbounded domain can be represented as a sum of two invariant subspaces. Their spectra correspond to a bounded and unbounded parts of a convex domain. On the base of this result we obtain a simple geometric criterion of the fundamental principle for an invariant subspace of analytic functions in a half-plane. It is formulated just in terms of the Krisvosheev condensation index for the sequence of exponents of the mentioned exponential monomials.
\end{abstract}

Keywords: invariant subspace, fundamental principle, exponential monomial, entire function, series of exponentials.

Mathematics Subject Classification: 30D10

\section{INTRODUCTION}

Let $\Lambda=\left\{\lambda_{k}, n_{k}\right\}_{k=1}^{\infty}$ be a sequence of different complex number $\lambda_{k}$ and of their multiplicities $n_{k}$. We assume that $\left|\lambda_{k}\right|$ are non-decreasing and $\left|\lambda_{k}\right| \rightarrow \infty, k \rightarrow \infty$. By $\Xi(\Lambda)$ we denote the set of limits of converging sequences of form $\left\{\bar{\lambda}_{k_{j}} /\left|\lambda_{k_{j}}\right|\right\}_{j=1}^{\infty}$, where $\bar{\lambda}$ stands for the complex conjugation. The set $\Xi(\Lambda)$ is closed and is a subset of the unit circumference $S(0,1)$. We introduce a family of exponential monomials

$$
\mathcal{E}(\Lambda)=\left\{z^{n} e^{\lambda_{k} z}\right\}_{k=1, n=0}^{\infty, n_{k}-1}
$$

Let $D \subset \mathbb{C}$ be a convex domain and

$$
H_{D}(\varphi)=\sup _{z \in D} \operatorname{Re}\left(z e^{-i \varphi}\right), \quad \varphi \in[0,2 \pi]
$$

A.S. Krivosheev, O.A. Krivosheeva, Invariant subspaces in half-Plane.

(C)Krivosheev A.S., Krivosheeva O.A. 2020.

Submitted April 5, 2020.

The research of the second co-author was supported by Russian Science Foundation (grant no. 18-11-00002). 
be its support function. We let

$$
J(D)=\left\{e^{i \varphi} \in S(0,1): H_{D}(\varphi)=+\infty\right\} .
$$

If $D$ is a bounded domain, then $J(D)=\emptyset$. In the case of an unbounded domain the following situations are possible:

1) $J(D)=S(0,1)$, that is, $D=\mathbb{C}$,

2) $D$ is a half-plane $\left\{z \in \mathbb{C}: \operatorname{Re}\left(z e^{-i \varphi}\right)<a\right\}$ and $J(D)=S(0,1) \backslash\left\{e^{i \varphi}\right\}$,

3) $D$ is a strip- $\left\{z \in \mathbb{C}: b<\operatorname{Re}\left(z e^{-i \varphi}\right)<a\right\}$ and $J(D)=S(0,1) \backslash\left\{e^{i \varphi}, e^{i \varphi+\pi}\right\}$,

4 ) in other case $J(D)$ is an arc on the unit circle leaning on an angle of opening at least $\pi$.

By the symbol int $J(D)$ we denote the interior of the set $J(D)$ in the topology of the circumference $S(0,1)$.

Let $H(D)$ be the space of functions analytic in a domain $D$ with the topology of uniform convergence on compact sets $K \subset D$, and let $W \subset H(D)$ be a non-trivial, that is, $W \neq$ $\{0\}, W \neq H(D)$, closed subspace invariant with respect to the differentiation operator. The spectrum of this operator in the subspace $W$ is an at most countable set $\left\{\lambda_{k}\right\}$ [1, Ch. II, Sect. 7]. Let $\Lambda=\left\{\lambda_{k}, n_{k}\right\}$ be a multiple spectrum of the differentiation operator in the subspace $W$. Then $\mathcal{E}(\Lambda)$ is the family of its eigenfunctions and adjoint functions in $W$. The subspace $W$ is said to admit the spectral synthesis if it coincides with the closure of linear span $W(\Lambda, D)$ of the system $\mathcal{E}(\Lambda)$ in the space $H(D)$. We mention that the problem of spectral synthesis was complete solved in works [2] and [3]. If $D$ is an unbounded convex domain, then identity $W=W(\Lambda, D)$ holds, that is, $W$ admits the spectral synthesis [3, Thm 8.2].

Particular cases of invariant subspaces are the spaces of solutions of linear homogeneous differential, difference and differential-difference equations both of finite and infinite orders, as well as of more general convolution equations and their systems.

A main problem in the theory of invariant subspace is the fundamental principle problem, that is, representing each function from $W$ by means of the series over the elements of the system $\mathcal{E}(\Lambda)$. The fundamental principle is said to hold in a subspace $W$ with the spectrum $\left\{\lambda_{k}, n_{k}\right\}$ if for each function $g \in W$ the representation holds:

$$
g(z)=\sum_{k=1, n=0}^{\infty, n_{k}-1} d_{k, n} z^{n} e^{\lambda_{k} z}, \quad z \in D,
$$

and this series converges uniformly on compact sets in $D$. This issue is called fundamental principle problem. The name "fundamental principle" arisen in relation with a particular case of the invariant subspace, namely, the space of solutions to a linear homogeneous differential equation with constant coefficients. It is known that each solution to such equation is a linear combination of elementary solutions, exponential monomials $z^{n} e^{\lambda_{k} z}$, with exponents being zeroes, probably, multiple, of a characteristic polynomial. The presence of such representation is called Euler fundamental principle.

By means of Laplace transform, the fundamental principle problem is reduced to a dual problem of multiple interpolation in the space of entire functions of exponential type. The study of both problems made first independently have a rich history. Its main milestones are reflected in works [4] and [5]. In the case of a bounded convex domain the fundamental principle problem was completely solved in works [5]-[8]. There was obtained a simple geometric criterion of the fundamental principle [8, Thm. 3.2] for invariant subspaces admitting the spectral synthesis, which was formulated only in terms of the Krivosheed condensation index $S_{\Lambda}$, which will be introduced below, of the maximal angular density of the sequence $\Lambda$ and of the length of the boundary of the domain $D$.

The situation with unbounded convex domains is much worse. In work [5], there was obtained a criterion of the fundamental principle for arbitrary convex domains. It however has two disadvantages. It involves some restriction for the multiplicity $n_{k}$ of the points $\lambda_{k}$. Moreover, it 
involves the following condition, which is equivalent to the validity of the fundamental principle. Namely, it requires the existence of a family of entire functions vanishing at the points $\lambda_{k}$ with the multiplicities at least $n_{k}$, the growth of which is close to a regular one and is related with $D$. There remained an open question under which conditions for $\Lambda$ and $D$ such family exists. The problem on constructing such family is rather complicated. Concerning unbounded domains, mostly only two particular cases were studied, namely, when $D$ was the plane or a half-plane.

A complete solution of the fundamental principle problem for non-trivial invariant subspaces of entire functions was obtained in work [9]. It was proved that the validity of the fundamental principle in each such subspace is equivalent to the finiteness of the condensation index $S_{\Lambda}$.

Invariant subspaces in a half-plane were studied in the case of a simple positive spectrum possessing a density. In work [10] this problem was solved completely for an arbitrary convex domain $D$. The solution was given in terms of simple geometric characteristics of the sequence $\Lambda$ and the domain $D$. It involves a principally new aspect. It turned out that in the case of a vertical half-plane, for the validity of the fundamental principle, the measurability of the sequence $\Lambda$ is not needed, and even the finiteness of its maximal density is not needed despite the support function of the half-plane is bounded in the positive direction. A necessary and sufficient condition in this situation is the vanishing of the characteristics $S_{\Lambda}$. In work [11] this result was extended for the case of invariant subspaces with an almost real spectrum $\Lambda$, that is, as $\Xi(\Lambda)=\{1\}$. We note that the result of work [11] is easily extended for the case of invariant subspaces with the spectrum $\Lambda$, for which $\Xi(\Lambda)$ is a one-point set.

The present work is devoted to studying nontrivial invariant subspaces with an arbitrary spectrum in a half-plane.

The work consists of four sections. In the second section we provide some preliminaries. In the third section we study the problem on decomposition of an invariant subspace into the sum of two invariant subspaces. We prove that an invariant subspace in each unbounded domain can be represented as a sum of two invariant subspaces. Their spectra correspond to a bounded and an unbounded parts of the convex domain.

In the last section we solve completely the fundamental principle problem for invariant subspaces in a half-plane. We obtain a simple geometric criterion for the fundamental principle, which is based only on the notion of the condensation index of a sequence forming the spectrum of an invariant subspace.

\section{PRELIMINARIES}

We begin with recalling some notions and facts related with an interpolating Leontiev function. Let $\Lambda=\left\{\lambda_{k}, n_{k}\right\}$ and $f$ be an entire function of exponential type, that is,

$$
\ln |f(\lambda)| \leqslant A+B|\lambda|, \quad \lambda \in \mathbb{C}, \quad A, B \geqslant 0 .
$$

We write $f(\Lambda)=0$ if $f$ vanishes at the points $\lambda_{k}$ with multiplicity at least $n_{k}$. An indicator of $f$ is the function

$$
h_{f}(\varphi)=\varlimsup_{t \rightarrow \infty} \frac{\ln \left|f\left(t e^{i \varphi}\right)\right|}{t}, \quad \varphi \in[0,2 \pi] .
$$

It coincides with the support function of some convex set $T \subset \mathbb{C}$ called an indicator diagram of $f$. By $\gamma(t, f)$ we denote the function associated with $f$ in the Borel sense [1, Ch. I, Sect. 5]. An adjoint diagram $K$ of the function $f$ is a convex hull of the set of singular points of $\gamma(t, f)$. Thus, $\gamma(t, f)$ is analytic outside some compact set $K$. By Polya theorem [1, Ch. I, Sect. 5, Thm. 5.4],

$$
h_{f}(\varphi)=H_{T}(\varphi)=H_{K}(-\varphi), \quad \varphi \in[0,2 \pi] .
$$

Therefore, $K$ is a compact set complex conjugate with the compact set $T$. 
Let $D$ be a convex domain, $g \in H(D), 0 \in K$, and $\sigma \in \mathbb{C}$ be such that the shift $K+\sigma$ of the adjoint diagram $K$ of the function $f$ lies in the domain $D$. An interpolating function for a function $g$ is [12, Ch. I, Sect. 2]

$$
\omega_{f}(\lambda, \sigma, g)=e^{-\sigma \lambda} \frac{1}{2 \pi i} \int_{\Omega} \gamma(t, f)\left(\int_{0}^{t} g(t+\sigma-\eta) e^{\lambda \eta} d \eta\right) d t,
$$

where $\Omega$ is a contour, that is, a simple closed continuous rectifiable curve enveloping the compact set $K$ and is located in the domain $D-\sigma$.

We are going to omit the restriction $0 \in K$. We choose an arbitrary point $w \in K$. An adjoint diagram of the function $f_{w}(z)=f(z) e^{-w z}$ coincides with the compact set $K_{w}=K-w$ containing the origin. Then by formula (2.2) we define the function $\omega_{f_{w}}(\lambda, \sigma, g)$ for all $\sigma \in \mathbb{C}$ such that the compact set $K_{w}+\sigma$ lies in the domain $D$.

Let us mention some properties of the function $\omega_{f_{w}}(\lambda, \sigma, g)$. It follows from (2.2) that this function is entire and linear in the third independent variable. Let $K(\varepsilon)=K+B(0, \varepsilon)$ be an $\varepsilon$-swelling of the compact set $K, \Omega(\varepsilon)=\partial(K(\varepsilon))-w$ and $\Omega_{\sigma}(\varepsilon)=\Omega(\varepsilon)+\sigma \subset G$. By (2.2) we have:

$$
\begin{aligned}
\left|\omega_{f_{w}}(\lambda, \sigma, g)\right| & \leqslant \frac{1}{2 \pi}\left|e^{-\sigma \lambda}\right| \max _{z \in \Omega(\varepsilon)}\left|e^{\lambda z}\right| \max _{z \in \Omega_{\sigma}(\varepsilon)}|g(z)| \int_{\Omega(\varepsilon)}\left|\gamma\left(t, f_{w}\right)\right||t||d t| \\
& \leqslant \tau_{\varepsilon} \exp \left(r H_{\Omega(\varepsilon)}(-\varphi)-\operatorname{Re}(\sigma \lambda)\right) \max _{z \in \Omega_{\sigma}(\varepsilon)}|g(z)| \int_{\partial K(\varepsilon)}|\gamma(t, f)||d t| \\
& =A(f, \varepsilon) \exp \left(r H_{K}(-\varphi)+\varepsilon r-\operatorname{Re}(w \lambda)-\operatorname{Re}(\sigma \lambda)\right) \sup _{z \in \Omega_{\sigma}(\varepsilon)}|g(z)|, \quad \lambda=r e^{i \varphi},
\end{aligned}
$$

where $A(f, \varepsilon)=(2 \pi)^{-1} \tau_{\varepsilon}(f) d_{\varepsilon}, d_{\varepsilon}$ is the diameter of the domain $K(\varepsilon)$ and $\tau_{\varepsilon}(f)$ is the latter integral. In view of identity (2.1), this implies that

$$
\left|\omega_{f_{w}}(\lambda, \sigma, g)\right| \leqslant A(f, \varepsilon) \exp \left(\left(h_{f}(\varphi)+\varepsilon\right) r-\operatorname{Re}((w+\sigma) \lambda)\right) \max _{z \in \Omega_{\sigma}(\varepsilon)}|g(z)| .
$$

for all $\lambda \in \mathbb{C}$.

Let us mention a main property of the interpolating function. Let $\Lambda=\left\{\lambda_{k}, n_{k}\right\}$ be a multiple zero set of the function $f$ and

$$
P(z)=\sum_{k=1}^{p} \sum_{n=0}^{n_{k}-1} a_{k, n} z^{n} e^{\lambda_{k} z}
$$

Then the identities hold [12, Ch. I, Sect. 2, Thm. 1.2.4]:

$$
\frac{1}{2 \pi i} \int_{\partial B\left(\lambda_{k}, b_{k}\right)} \frac{\omega_{f_{w}}(\lambda, \sigma, P)}{f_{w}(\lambda)} e^{\lambda z} d \lambda=\sum_{n=0}^{n_{k}-1} a_{k, n} z^{n} e^{\lambda_{k} z}, \quad \sigma \in \mathbb{C}, \quad k=\overline{1, p}
$$

where $\partial B\left(\lambda_{k}, b_{k}\right)$ is a circumference containing no points $\lambda_{s}, s \neq k$. The following statements are particular cases of respectively Theorems 2.1.1 and 2.1.2 from book [12].

Lemma 2.1. Let $\Lambda=\left\{\lambda_{k}, n_{k}\right\}, D$ be a convex domain and the system $\mathcal{E}(\Lambda)$ be incomplete in the space $H(D)$. Assume that

$$
g(z)=\lim _{\mu \rightarrow \infty} P_{\mu}(z), \quad P_{\mu}(z)=\sum_{k=1}^{\mu} \sum_{n=0}^{n_{k}-1} a_{k, n, \mu} z^{n} e^{\lambda_{k} z}
$$


where the convergence is uniform on compact sets in the domain D. Then there exist the limits

$$
a_{k, n}=\lim _{\mu \rightarrow \infty} a_{k, n, \mu}, \quad n=\overline{0, n_{k}-1}, \quad k \geqslant 1 .
$$

Lemma 2.2. Let $\Lambda=\left\{\lambda_{k}, n_{k}\right\}, D$ be a convex domain and the system $\mathcal{E}(\Lambda)$ be incomplete in the space $H(D)$. Assume that 2.5 holds and

$$
g(z)=)=\lim _{\mu \rightarrow \infty} Q_{\mu}(z), \quad Q_{\mu}(z)=\sum_{k=1}^{\mu} \sum_{n=0}^{n_{k}-1} b_{k, n, \mu} z^{n} e^{\lambda_{k} z}
$$

where the convergence is uniform on compact sets in the domain D. Then

$$
\lim _{\mu \rightarrow \infty} a_{k, n, \mu}=\lim _{\mu \rightarrow \infty} b_{k, n, \mu}, \quad n=\overline{0, n_{k}-1}, \quad k \geqslant 1 .
$$

We introduce some more notions and notations. Let $\Lambda=\left\{\lambda_{k}, n_{k}\right\}, n(r, \Lambda)$ be the number of the points $\lambda_{k}$ (taken according their multiplicities $n_{k}$ ) located in an open ball $B(0, r)$ and

$$
\bar{n}(\Lambda)=\lim _{r \rightarrow+\infty} \frac{n(r, \Lambda)}{r}
$$

be the upper density of the sequence $\Lambda$. According the well-known Lindelöf theorem [13, Ch. I, Sect. 11, Thm. 15], one has $\bar{n}(\Lambda)<+\infty$ if and only if there exists an entire function $f$ of exponential type such that $f(\Lambda)=0$.

Let $U=\left\{U_{m}\right\}_{m=1}^{\infty}$ be a partition of the sequence $\left\{\lambda_{k}\right\}$ into finite subgroups, that is, $U_{m}$ consists in finitely many points $\lambda_{k}, U_{m} \cap U_{l}=\emptyset, l \neq m$, and $\cup_{m} U_{m}=\left\{\lambda_{k}\right\}$. We let

$$
d_{\Lambda}(U)=\varlimsup_{m \rightarrow \infty} \max _{\lambda_{k}, \lambda_{v}, \lambda_{j} \in U_{m}} \frac{\left|\lambda_{k}-\lambda_{v}\right|}{\left|\lambda_{j}\right|} .
$$

Following [7], we introduce the condensation index:

$$
S_{\Lambda}(U)=\lim _{\delta \rightarrow 0} \lim _{m \rightarrow \infty} \min _{\lambda_{k} \in U_{m}} \frac{\ln \left|q_{\Lambda, U}^{m, k}\left(\lambda_{k}, \delta\right)\right|}{\left|\lambda_{k}\right|}, \quad q_{\Lambda, U}^{m, k}(z, \delta)=\prod_{\lambda_{v} \in B\left(\lambda_{k}, \delta\left|\lambda_{k}\right|\right) \backslash U_{m}}\left(\frac{z-\lambda_{v}}{3 \delta\left|\lambda_{v}\right|}\right)^{n_{v}} .
$$

First the condensation index was introduced in work [5] for the trivial partition, that is, when each group $U_{m}$ consists in one point. In the case when the partition $U$ is trivial, the quantity $S_{\Lambda}(U)$ coincides with the quantity $S_{\Lambda}$ introduced in [5]. This is why in this case we write $S_{\Lambda}$ instead of $S_{\Lambda}(U)$.

The next two statements were proved in work [9], see Theorems 2.1 and 5.1 in the cited work. In the second statement the main idea of introducing quantity $S_{\Lambda}(U)$ is clarified.

Theorem 2.3. Let $\Lambda=\left\{\lambda_{k}, n_{k}\right\}$ and $\bar{n}(\Lambda)<+\infty$. Then for each $d>0$ there exists a partition $U$ of the sequence $\Lambda$ such that $S_{\Lambda}(U)>-\infty$ and $d_{\Lambda}(U)<d$.

Theorem 2.4. Let $f$ be an entire function of exponential type, $\Lambda=\left\{\lambda_{k}, n_{k}\right\}$ be its multiple zero set, $U=\left\{U_{m}\right\}$ be the partition of $\Lambda$, for which $S_{\Lambda}(U)>-\infty$ and $d_{\Lambda}(U)<+\infty$. Then there exist positive numbers $\left\{\gamma_{k}\right\}_{k}^{\infty}$ such that

$$
\varlimsup_{m \rightarrow \infty} \max _{\lambda_{k}, \lambda_{v} \in U_{m}} \frac{\gamma_{k}}{\left|\lambda_{v}\right|}<+\infty
$$

the sets $B_{m}=\bigcup_{\lambda_{k} \in U_{m}} B\left(\lambda_{k}, \gamma_{k}\right), m \geqslant 1$, are mutually disjoint and for each $\beta \in(0,1)$ there exist $a, a_{1}>0$ such that

$$
\ln |f(z)| \geqslant-a_{1}-a|z|, \quad z \in \partial B_{m}(\beta), \quad m \geqslant 1, \quad B_{m}(\beta)=\bigcup_{\lambda_{k} \in U_{m}} B\left(\lambda_{k}, \beta \gamma_{k}\right) .
$$




\section{DECOMPOSITION OF INVARIANT SUBSPACES}

Let $D$ be an unbounded convex domain and $W$ be a nontrivial closed subspace in $H(D)$ invariant with respect to the differentiation. As it has been mentioned above, in this case the space $W$ admits the spectral synthesis, that is, the identity $W=W(\Lambda, D)$ holds, where $\Lambda$ is the multiple spectrum of the differentiation operator in the subspace $W$.

The non-triviality of the subspace $W(\Lambda, D)$ means that the system $\mathcal{E}(\Lambda)$ is incomplete in the space $H(D)$. In view of this, in order to study invariant subspaces $W \subset H(D)$ in an unbounded convex domain, it is sufficient to consider the case when $W=W(\Lambda, D)$ and $\mathcal{E}(\Lambda)$ is incomplete $H(D)$.

The system $\mathcal{E}(\Lambda)$ is incomplete in the space $H(D)$ if and only if [1, Ch. I, Sect. 7, Thm. 7.2; Sect. 5, Thm. 5.2] there there exists an entire function $f$ of exponential type such that $f(\Lambda)=0$ and some shift $K+\sigma$ of its adjoint diagram $K$ is located in the domain $D$.

The issue on completeness of the system $\mathcal{E}(\Lambda)$ in the space $H(D)$ is resolved easily only in the case, when the domain $D$ can not be moved into any strip. This is a so-called large convex domain. It contains some shift of each convex set. Therefore, in view of the Lindelöf theorem, for a large convex domain, the system $\mathcal{E}(\Lambda)$ is incomplete in $H(D)$ if and only if $\bar{n}(\Lambda)<+\infty$.

In the case when $D$ is located in some strip, a simple criterion of the completeness of the system $\mathcal{E}(\Lambda)$ was obtained in works [14], [15]. It is formulated in terms of a logarithmic blockdensity of the sequence $\Lambda$.

Let $D$ be an unbounded convex domain. By $J_{0}(D)$ we denote the subset of the set $J(D)$ consisting of all points $e^{i \varphi}$ such that

$$
\left\{e^{i \alpha}: \alpha \in(\varphi-\pi / 2, \varphi+\pi / 2)\right\} \subset J(D) .
$$

By definition, $J_{0}(D)$ is a closed subset of the unit circumference $S(0,1)$. If $D=\mathbb{C}$, then $J_{0}(D)=S(0,1)$. If $D$ is a half-plane $\left\{z \in \mathbb{C}: \operatorname{Re}\left(z e^{-i \varphi}\right)<a\right\}$, then $J_{0}(D)=\left\{e^{i \alpha}: \alpha \in\right.$ $[-\varphi-\pi / 2,-\varphi+\pi / 2]\}$. If $D$ is located in the strip $\left\{z \in \mathbb{C}: b<\operatorname{Re}\left(z e^{-i \varphi}\right)<a\right\}$, then either $J_{0}(D)$ consists of two points $\left\{e^{i(\varphi-\pi / 2)}, e^{i(\varphi+\pi / 2)}\right\}$ (if $D$ coincides with this or a smaller strip) or coincides with one of these points (otherwise). In other cases $J_{0}(D)$ is an $\operatorname{arc}\left\{e^{i \alpha}: \alpha \in\left[\varphi_{1}, \varphi_{2}\right]\right\}$ on the unit circumference leaning on an angle of opening strictly less than $\pi$.

Thus, if the domain $D$ is not a strip, then for some $\varphi_{1}, \varphi_{2}$ such that $\pi \leqslant \varphi_{2}-\varphi_{1} \leqslant 2 \pi$ the identities hold:

$$
J(D)=\left\{e^{i \varphi}: \varphi \in I\right\}, \quad J_{0}(D)=\left\{e^{i \varphi}: \varphi \in\left[\varphi_{1}+\pi / 2, \varphi_{2}-\pi / 2\right]\right\},
$$

where $I$ is a segment, an interval or a semi-interval with the end-points at $\varphi_{1}, \varphi_{2}$. If $\varphi_{2}-\varphi_{1}=\pi$, then the segment $\left[\varphi_{1}+\pi / 2, \varphi_{2}-\pi / 2\right]$ degenerates into a point. For instance, this happens if $D$ is a domain enveloped by the parabola or if $D$ is located inside a strip but does not coincides with it. The identity $\varphi_{2}-\varphi_{1}=2 \pi$ is realized only in the case when $D$ is a half-plane.

Lemma 3.1. Let $D$ be an unbounded convex domain and $M$ be a subset of $D$. Then for each $e^{i \varphi} \in J_{0}(D)$ and $t>0$ the shift $M+t e^{i \varphi}$ is located in $D$.

Proof. Let $e^{i \varphi} \in J_{0}(D), t>0$ and $z \in M$. According the definition of $J_{0}(D)$, we have

$$
\operatorname{Re}\left(\left(z+t e^{i \varphi}\right) e^{-i \alpha}\right)<+\infty=H_{D}(\alpha), \quad \alpha \in(\varphi-\pi / 2, \varphi+\pi / 2) .
$$

Moreover, for each $\alpha \in[\varphi+\pi / 2, \varphi+3 \pi / 2]$ we get:

$$
\operatorname{Re}\left(\left(z+t e^{i \varphi}\right) e^{-i \alpha}\right)=\operatorname{Re}\left(z e^{-i \alpha}\right)+\operatorname{Re}\left(t e^{i \varphi} e^{-i \alpha}\right) \leqslant \operatorname{Re}\left(z e^{-i \alpha}\right)<H_{D}(\alpha) .
$$

Thus,

$$
\operatorname{Re}\left(\left(z+t e^{i \varphi}\right) e^{-i \alpha}\right)<H_{D}(\alpha), \quad \alpha \in[0,2 \pi] .
$$

This means that $z+t e^{i \varphi} \in D$. The proof is complete. 
Lemma 3.2. Let $D$ be an unbounded convex domain, $0<\alpha_{2}-\alpha_{1}<\pi$ and

$$
\Xi_{0}=\left\{e^{i \varphi}: \varphi \in\left[\alpha_{1}, \alpha_{2}\right]\right\} \subseteq \operatorname{int} J(D) .
$$

Then there exists $e^{i \varphi_{0}} \in J_{0}(D)$ such that for all $R, C>0$ there exists $t_{0}>0$ satisfying the condition

$$
\operatorname{Re}\left(\left(t e^{i \varphi_{0}}-z\right) e^{i \alpha}\right) \geqslant C, \quad e^{-i \alpha} \in \Xi_{0}, \quad|z| \leqslant R, \quad t \geqslant t_{0} .
$$

Proof. Since $\Xi_{0} \in$ int $J(D)$ and $0<\alpha_{2}-\alpha_{1}<\pi$, according to the definition of the set $J_{0}(D)$ there exists $e^{i \varphi_{0}} \in J_{0}(D)$ such that $\Xi_{0} \subset\left\{e^{i \alpha}: \alpha \in\left(\varphi_{0}-\pi / 2, \varphi_{0}+\pi / 2\right)\right\}$. Then for some $\delta_{0}>0$ the embedding holds:

$$
\Xi_{0} \subset\left\{e^{i \alpha}: \alpha \in\left[\varphi_{0}-\pi / 2+\delta_{0}, \varphi_{0}+\pi / 2-\delta_{0}\right]\right\} .
$$

Let $R, C>0$. By (3.1) there exists $c>0$ such that

$$
\operatorname{Re}\left(\left(t e^{i \varphi_{0}}-z\right) e^{i \alpha}\right) \geqslant \operatorname{Re}\left(t e^{i \varphi_{0}} e^{i \alpha}\right)-R \geqslant t c-R, \quad e^{-i \alpha} \in \Xi_{0}, \quad|z| \leqslant R .
$$

For $t_{0}=(R+C) c^{-1}$ this implies the desired inequality. The proof is complete.

Now we are in position to formulate the results on decomposition of invariant subspaces.

Lemma 3.3. Let $\Lambda=\left\{\lambda_{k}, n_{k}\right\}, D$ be a convex domain and the system $\mathcal{E}(\Lambda)$ be incomplete in $H(D)$. For each $m \geqslant 1$ and each function $g \in W(\Lambda, D)$ the representation holds $g=g_{1}+g_{2}$, where $g_{1} \in W\left(\Lambda_{1}, \mathbb{C}\right)$ and $g_{2} \in W\left(\Lambda_{2}, D\right)$,

$$
\Lambda_{1}=\left\{\lambda_{k}, n_{k}\right\}_{k=1}^{m}, \quad \Lambda_{2}=\left\{\lambda_{k}, n_{k}\right\}_{k=m+1}^{\infty},
$$

and $W\left(\Lambda_{1}, \mathbb{C}\right)$ is the space of exponential polynomials

$$
P(z)=\sum_{k=1}^{m} \sum_{n=0}^{n_{k}-1} a_{k, n} z^{n} e^{\lambda_{k} z}, \quad a_{k, n} \in \mathbb{C}, \quad n=\overline{0, n_{k}-1}, \quad k=\overline{1, m} .
$$

Proof. Let $m \geqslant 1$. Since $g \in W(\Lambda, D)$, then

$$
g(z)=\lim _{\mu \rightarrow \infty} P_{\mu}(z), \quad P_{\mu}(z)=\sum_{k=1}^{\mu} \sum_{n=0}^{n_{k}-1} a_{k, n, \mu} z^{n} e^{\lambda_{k} z}
$$

and the convergence is uniform on compact sets in D. By Lemma 2.1 there exist the limits

$$
a_{k, n}=\lim _{\mu \rightarrow \infty} a_{k, n, \mu}, \quad n=\overline{0, n_{k}-1}, \quad k \geqslant 1 .
$$

Therefore, the sequence of polynomials

$$
P_{\mu, 1}(z)=\sum_{k=1}^{m} \sum_{n=0}^{n_{k}-1} a_{k, n, \mu} z^{n} e^{\lambda_{k} z}, \quad \mu \geqslant 1
$$

converge uniformly on compact sets in the plane to a polynomial

$$
g_{1}(z)=\sum_{k=1}^{m} \sum_{n=0}^{n_{k}-1} a_{k, n} z^{n} e^{\lambda_{k} z}
$$

Then by $(3.2)$ the sequence of polynomials

$$
P_{\mu, 2}(z)=\sum_{k=m+1}^{\mu} \sum_{n=0}^{n_{k}-1} a_{k, n, \mu} z^{n} e^{\lambda_{k} z}, \quad \mu \geqslant m+1,
$$

converges to the function $g_{2}=g-g_{1}$ uniformly on compact sets in the domain $D$. This is why $g_{2} \in W\left(\Lambda_{2}, D\right)$. The proof is complete. 
Let $\Lambda=\left\{\lambda_{k}, n_{k}\right\}, \Lambda_{1}=\left\{\xi_{p}, m_{p}\right\}$ and $\Lambda_{2}=\left\{\varsigma_{j}, l_{j}\right\}$. We write $\Lambda=\Lambda_{1} \cup \Lambda_{2}$ if for each $k \geqslant 1$ there exists $p \geqslant 1$ such that $\lambda_{k}=\xi_{p}$ and $n_{k}=m_{p}$ or there exists $j \geqslant 1$ such that $\lambda_{k}=\varsigma_{j}$ and $n_{k}=l_{j}$.

Theorem 3.4. Let $\Lambda=\left\{\lambda_{k}, n_{k}\right\}, D$ be a convex domain and the system $\mathcal{E}(\Lambda)$ is incomplete in $H(D)$. Then there exist sequences $\Lambda_{1}$ and $\Lambda_{2}$ such that

$$
\Lambda=\Lambda_{1} \cup \Lambda_{2}, \quad \Xi\left(\Lambda_{2}\right) \subset S(0,1) \backslash \operatorname{int} J(D),
$$

and for each function $g \in W(\Lambda, D)$ the representation $g=g_{1}+g_{2}$ holds, where $g_{1} \in W\left(\Lambda_{1}, \mathbb{C}\right)$ and $g_{2} \in W\left(\Lambda_{2}, D\right)$. In particular, $\Lambda_{1}=\emptyset$ and $g_{1}=0$ if $\Xi(\Lambda) \cap \operatorname{int} J(D)=\emptyset$ and $\Lambda_{2}=\emptyset$ and $g_{2}=0$ if $\Xi(\Lambda) \subset \operatorname{int} J(D)$.

Proof. Let $g \in W(\Lambda, D)$. First we consider the case

$$
\Xi(\Lambda) \cap \operatorname{int} J(D)=\emptyset .
$$

We let $\Lambda_{1}=\emptyset, g_{1}=0, \Lambda_{2}=\Lambda$ and $g_{2}=g$. Then $g_{2} \in W\left(\Lambda_{2}, D\right)=W(\Lambda, D)$. Hence, in this case the statement of theorem holds true.

Suppose that $\Xi(\Lambda) \cap \operatorname{int} J(D) \neq \emptyset$. We choose sequences $\left\{\alpha_{1, l}^{s}\right\},\left\{\alpha_{2, l}^{s}\right\}, s=1,2$, such that

$$
\begin{aligned}
& \alpha_{2, l}^{s}-\alpha_{1, l}^{s}<\pi, \quad \alpha_{1, l+1}^{s}<\alpha_{1, l}^{s}<\alpha_{2, l}^{s}<\alpha_{2, l+1}^{s}, \quad l \geqslant 1, \\
& \Xi_{s, l}=\left\{e^{i \varphi}: \varphi \in\left[\alpha_{1, l}^{s}, \alpha_{2, l}^{s}\right]\right\} \subset \operatorname{int} J(D), \quad s=1,2, \\
& \bigcup_{l=1}^{\infty}\left(\Xi_{1, l} \cup \Xi_{2, l}\right)=\operatorname{int} J(D) .
\end{aligned}
$$

By our assumptions, the system $\mathcal{E}(\Lambda)$ is incomplete in the space $H(D)$. Then there exists an entire function $f$ of exponential type such that $f(\Lambda)=0$ and some shift $K+w_{0}$ of its adjoint diagram $K$ is located in the domain $D$.

Let $\Lambda^{1}$ be a multiple zero set of the function $f$. Since $f$ is of exponential type, then by Lindelöf theorem we have: $\bar{n}\left(\Lambda^{1}\right)<+\infty$. Then by Theorem 2.3 for each $d>0$ there exists a partition $U(d)=\left\{U_{m}(d)\right\}$ of the sequence $\Lambda^{1}$ such that inequalities $S_{\Lambda^{1}}(U(d))>-\infty, d_{\Lambda^{1}}(U(d))<d$ hold.

We fix $l \geqslant 1$. We form a subsequence $\left\{U_{m(1, l, j)}(d)\right\}$ of all groups $U_{m}(d)$, each of them contains a point $\lambda_{k}=r_{k} e^{i \varphi_{k}}$ such that $e^{-i \varphi_{k}} \in \Xi_{1, l}$, and also a subsequence $\left\{U_{m(2, l, j)}(d)\right\}$ of all groups $U_{m}(d)$, each of them contains a point $\lambda_{k}=r_{k} e^{i \varphi_{k}}$ such that $e^{-i \varphi_{k}} \in \Xi_{2, l}$ and $e^{-i \varphi_{p}} \in \Xi_{1, l}$ for all $\lambda_{p}=r_{p} e^{i \varphi_{p}} \in U_{m(2, l, j)}(d)$; in some case the set $\left\{U_{m(2, l, j)}(d)\right\}$ can be empty. We choose $d_{l} \in(0,1 / 2)$ and $j_{l}$ so that $e^{-i \varphi_{k}} \in \Xi_{s, l+1}$ for all $\lambda_{k} \in U_{m(s, l, j)}\left(d_{l}\right), j \geqslant j_{l}$ and $s=1,2$.

By Theorem 2.4 we find positive numbers $\gamma_{k}=\gamma_{k, l}, k \geqslant 1$, such that (2.6) holds and the sets $B_{m, l}=\cup_{\lambda_{k} \in U_{m}\left(d_{l}\right)} B\left(\lambda_{k}, \gamma_{k, l}\right), m \geqslant 1$, are mutually disjoint and also for each number $\beta \in(0,1)$ we find $a=a(\beta), a_{1}=a_{1}(\beta)>0$ such that $(2.7)$ holds true.

Increasing the index $j_{l}$ if needed, we find $\beta_{l} \in(0,1 / 2)$ such that for all $j \geqslant j_{l}$ and $z=r e^{i \varphi} \in$ $B_{m(s, l, j)}\left(\beta_{l}\right)$ the belonging holds: $e^{-i \varphi} \in \Xi_{s, l+2}, s=1,2$.

Let us define the sets $B_{s, l, p}$. As $B_{s, 1, p}$ we take all sets $B_{m(s, 1, j)}\left(\beta_{1}\right)$. Let $l>1$. As $B_{s, l, p}$ we take all sets

$$
B_{m(s, l, j)}\left(\beta_{l}\right) \backslash\left(\bigcup_{\eta=1}^{2} \bigcup_{\nu=1}^{l-1} \bigcup_{\mu \geqslant 1} B_{\eta, \nu, \mu}\right),
$$

each of them contains at least one point $\lambda_{k}$.

We observe that the sets $B_{s, l, p}$ are mutually disjoint and each of them contains at least one point $\lambda_{k}$, and each point $\lambda_{k}=r_{k} e^{i \varphi_{k}}$ is such that $e^{-i \varphi_{k}} \in \operatorname{int} J(D)$ and belongs just to one of the sets $B_{s, l, p}$. 
We let

By (2.7) we have

$$
A_{l}=\max _{1 \leqslant \nu \leqslant l} a\left(\beta_{\nu}\right), \quad A_{1, l}=\max _{1 \leqslant \nu \leqslant l} a_{1}\left(\beta_{\nu}\right)
$$

$$
\ln |f(z)| \geqslant-A_{1, l}-A_{l}|z|, \quad z \in \partial B_{s, l, p}, \quad p, l \geqslant 1, \quad s=1,2 .
$$

Since $g \in W(\Lambda, D)$, then

$$
g(z)=\lim _{\mu \rightarrow \infty} P_{\mu}(z), \quad P_{\mu}(z)=\sum_{k=1}^{\mu} \sum_{n=0}^{n_{k}-1} a_{k, n, \mu} z^{n} e^{\lambda_{k} z}
$$

and the convergence is uniform on compact sets in the domain $D$. Let $w \in K$. By formula (2.2) we define an interpolating function $\omega_{f_{w}}\left(\lambda, \sigma, P_{\mu}\right)$ for all $\mu \geqslant 1$ and $\sigma \in \mathbb{C}$. We let $a_{k, n, \mu}=0$, $k>\mu$. It follows from 2.4 that

$$
\frac{1}{2 \pi i} \int_{\partial B\left(\lambda_{k}, b_{k}\right)} \frac{\omega_{f_{w}}\left(\lambda, \sigma, P_{\mu}\right)}{f_{w}(\lambda)} e^{\lambda z} d \lambda=\sum_{n=0}^{n_{k}-1} a_{k, n, \mu} z^{n} e^{\lambda_{k} z}, \quad \sigma \in \mathbb{C}, \quad k \geqslant 1 .
$$

We fix $l \geqslant 1$. By the theorem on residues we obtain:

$$
\int_{\partial B_{s, l, p}} \frac{\omega_{f_{w}}\left(\lambda, \sigma, P_{\mu}\right)}{f_{w}(\lambda)} e^{\lambda z} d \lambda=\sum_{\lambda_{k} \in B_{s, l, p}} a_{k, n, \mu} z^{n} e^{\lambda_{k} z}, \quad p \geqslant 1, \quad s=1,2 .
$$

Let

$$
h-1=\max _{\varphi \in[0,2 \pi]} h_{f}(\varphi)
$$

$s=1,2$, and $\varepsilon>0$. Since $P_{\mu}$ is an entire function, by (2.3), (3.3) and the definition of $f_{w}$ we get:

$$
\begin{aligned}
\left|\int_{\partial B_{s, l, p}} \frac{\omega_{f_{w}}\left(\lambda, \sigma, P_{\mu}\right)}{f_{w}(\lambda)} e^{\lambda z} d \lambda\right| \leqslant & A(f, \varepsilon) b_{s, l, p} \max _{\lambda \in \partial B_{s, l, p}}\left(\frac{\exp (|\lambda| h-\operatorname{Re}((w+\sigma-z) \lambda))}{\exp \left(-A_{1, l}-A_{l}|\lambda|-\operatorname{Re} w\right)}\right) \max _{x \in \Omega_{\sigma}(\varepsilon)}\left|P_{\mu}(x)\right| \\
= & A(f, \varepsilon) b_{s, l, p} e^{A_{1, l}} \max _{x \in \Omega_{\sigma}(\varepsilon)}\left|P_{\mu}(x)\right| \\
& \cdot \max _{\lambda \in \partial B_{s, l, p}} \exp \left(|\lambda|\left(h+A_{l}\right)-\operatorname{Re}((\sigma-z) \lambda)\right),
\end{aligned}
$$

where $b_{s, l, p}$ is the length of the boundary $\partial B_{s, l, p}, \sigma \in \mathbb{C}$ and $p \geqslant 1$.

For each $p \geqslant 1$ we choose a point $\lambda_{k(s, l, p)} \in B_{s, l, p}$. We note that the boundary $\partial B_{s, l, p}$ consists of two arcs on the circumferences $S\left(\lambda_{k}, \beta_{\nu} \gamma_{k}\right), \lambda_{k} \in B_{s, \nu, p}, \nu \leqslant l$, which have nonempty intersection with the circumferences $S\left(\lambda_{k}, \beta_{l} \gamma_{k}\right), \lambda_{k} \in B_{s, l, p}$. This is why in view of the inequalities $\bar{n}\left(\Lambda^{1}\right)<+\infty, d_{\Lambda^{1}}\left(U^{1}\left(d_{0}\right)\right)<+\infty$ and (2.6) there exist numbers $c_{l}, c_{0, l}>0$ such that

$$
b_{s, l, p} \leqslant c_{0, l}\left|\lambda_{k(s, l, p)}\right|^{2} \leqslant c_{l} e^{\left|\lambda_{k(s, l, p)}\right|}, \quad p \geqslant 1, \quad s=1,2 .
$$

Let $l \geqslant 1$. According the definition of the number $\beta_{l}$, there exists a number $p_{l}$ such that for all $p \geqslant p_{l}, s=1,2$ and $z=r e^{i \varphi} \in B_{s, l, p}$ the belonging holds: $e^{-i \varphi} \in \Xi_{s, l+2}$. By Lemma 3.2 , there exists $e^{i \varphi_{0}} \in J_{0}(D)$ such that for each $R>0$ there exists $t_{l}>0$ satisfying the condition:

$$
\operatorname{Re}\left(\left(t_{l} e^{i \varphi_{s, l}}-z\right) \lambda\right) \geqslant\left(h+A_{l}+|w|+\left|w_{0}\right|+4\right)|\lambda|, \quad \lambda \in \partial B_{s, l, p}, \quad p \geqslant p_{l}, \quad|z| \leqslant R .
$$

We let $\sigma_{s, l}=w+w_{0}+t_{l} e^{i \varphi_{s, l}}$ and take $p \geqslant p_{l}$ and $|z| \leqslant R$. Then by (3.5) we have:

$$
\left|\int_{\partial B_{s, l, p}} \frac{\omega_{f_{w}}\left(\lambda, \sigma_{s, l}, P_{\mu}\right)}{f_{w}(\lambda)} e^{\lambda z} d \lambda\right| \leqslant A(f, \varepsilon) e^{A_{1, l}} \max _{\mu \in \Omega_{\sigma_{s, l}}}(\varepsilon)\left|P_{\mu}(\mu)\right| b_{s, l, p} \max _{\lambda \in \partial B_{s, l, p}} \exp (-4|\lambda|) \text {. }
$$


Since $d_{l}, \beta_{l} \in(0,1 / 2)$, then increasing the index $p_{l}$ if needed, we can suppose that

$$
-4|\lambda| \leqslant-2\left|\lambda_{k(s, l, p)}\right|, \quad \lambda \in \partial B_{s, l, p}, \quad p \geqslant p_{l} .
$$

This is why in view of 3.5 we obtain:

$$
\left|\int_{\partial B_{s, l, p}} \frac{\omega_{f_{w}}\left(\lambda, \sigma_{s, l}, P_{\mu}\right)}{f_{w}(\lambda)} e^{\lambda z} d \lambda\right| \leqslant A(\varepsilon, l) \max _{\mu \in \Omega_{\sigma_{s, l}}}(\varepsilon)\left|P_{\mu}(\mu)\right| e^{-\left|\lambda_{k(s, l, p)}\right|}, \quad p \geqslant p_{l}, \quad|z| \leqslant R,
$$

where $A(\varepsilon, l)=A(f, \varepsilon) e^{A_{1, l}} c_{l}$. We have:

$$
\Omega_{\sigma_{s, l}}(\varepsilon)=\Omega(\varepsilon)+\sigma_{s, l}=\partial(K(\varepsilon))-w+\sigma_{s, l}=\partial(K(\varepsilon))+w_{0}+t_{l} e^{i \varphi_{s, l}} .
$$

The compact set $K+w_{0}$ is located in the domain $D$. This is why according Lemma 3.1, for some number $\varepsilon_{l}>0$, the compact set $\Omega_{\sigma_{s, l}}\left(\varepsilon_{l}\right)$ is also located in the domain $D$. The sequence $P_{\mu}$ converges uniformly on this compact set. Therefore,

$$
\left|\int_{\partial B_{s, l, p}} \frac{\omega_{f_{w}}\left(\lambda, \sigma_{s, l}, P_{\mu}\right)}{f_{w}(\lambda)} e^{\lambda z} d \lambda\right| \leqslant A\left(\varepsilon_{l}, l\right) B(R, l) e^{-\left|\lambda_{k(s, l, p)}\right|}, \quad p \geqslant p_{l}, \quad|z| \leqslant R,
$$

We choose a number $p_{l}(R)$ such that

$$
\left|\int_{\partial B_{s, l, p}} \frac{\omega_{f_{w}}\left(\lambda, \sigma_{s, l}, P_{\mu}\right)}{f_{w}(\lambda)} e^{\lambda z} d \lambda\right| \leqslant e^{-\left|\lambda_{k(s, l, p)}\right| / 2}, \quad p \geqslant p_{l}(R), \quad|z| \leqslant R .
$$

We can suppose that the function $p_{l}(R)$ is non-decreasing.

Let $\mu \geqslant 1$. We represent polynomials $P_{\mu}$ as

$$
\begin{aligned}
& P_{\mu}(z)=P_{\mu, 1}(z)+P_{\mu, 2}(z), \quad \mu \geqslant 1, \quad P_{\mu, 1}(z)=\sum_{k=1}^{\mu} \sum_{n=0}^{n_{k}-1} a_{k, n, \mu, 1} z^{n} e^{\lambda_{k} z} \\
& a_{k, n, \mu, 1}=a_{k, n, \mu}, \quad n=\overline{0, n_{k}-1}, \quad \lambda_{k} \in B_{s, l, p}, \quad s=1,2, \quad l \geqslant 1, \quad p \geqslant p_{l}(l), \\
& a_{k, n, \mu, 1}=0, \quad n=\overline{0, n_{k}-1}, \quad \lambda_{k} \in B_{s, l, p}, \quad s=1,2, \quad l \geqslant 1, \quad p \geqslant p_{l}(l) .
\end{aligned}
$$

In view of (3.4), the definition of the polynomial $P_{\mu, 1}$ and of the sets $B_{s, l, p}$ and since the latter are mutually disjoint, we obtain:

$$
P_{\mu, 1}(z)=\sum_{l=1}^{\infty} \sum_{p=p_{l}(l)}^{\infty} \frac{1}{2 \pi i} \int_{\partial B_{s, l, p}} \frac{\omega_{f_{w}}\left(\lambda, \sigma_{s, l}, P_{\mu}\right)}{f_{w}(\lambda)} e^{\lambda z} d \lambda, \quad \mu \geqslant 1 .
$$

We note that the latter sums contain only finitely many non-zero terms. Let $m \geqslant 1$. By (3.6) and (3.7) we have:

$$
\left|P_{\mu, 1}(z)\right| \leqslant \sum_{l=1}^{m-1} \sum_{p=p_{l}(l)}^{p_{l}(m)-1} A\left(\varepsilon_{l}, l\right) B(m, l)+\sum_{k=1}^{\infty} e^{-\left|\lambda_{k}\right| / 2}, \quad|z| \leqslant m, \quad \mu \geqslant 1 .
$$

Since $\bar{n}\left(\Lambda^{1}\right)<+\infty$, the latter series converges. Thus, the sequence of the functions $\left\{\left|P_{\mu, 1}\right|\right\}$ is uniformly bounded on each compact set in the plane. Applying the Montel's theorem, we find a subsequence $\left\{P_{\mu_{j}, 1}\right\}_{j=1}^{\infty}$ converging uniformly on each compact set in the plane. Let

$$
g_{1}, 0(z)=\lim _{j \rightarrow \infty} P_{\mu_{j}, 1}(z), \quad z \in \mathbb{C} .
$$

Since the sequence $\left\{P_{\mu}\right\}$ converges uniformly on compact sets in $D$, then $P_{\mu_{j}, 2}=P_{\mu_{j}}-P_{\mu_{j}, 1}$ also converges uniformly on compact sets in $D$ to some function $g_{2,0}$. It is obvious that $g=g_{1,0}+g_{2,0}$. 
By construction, $g_{1,0} \in W\left(\Lambda_{1,0}, \mathbb{C}\right)$, where $\Lambda_{1,0}$ is a sequence of all pairs $\lambda_{k}, n_{k}$ such that $\lambda_{k} \in B_{s, l, p}, s=1,2, l \geqslant 1, p \geqslant p_{l}(l)$. Let $\Lambda_{2,0}$ be a sequence completing $\Lambda_{1,0}$ to $\Lambda$, that is, $\Lambda=\Lambda_{1,0} \cup \Lambda_{2,0}$. Then $g_{2} \in W\left(\Lambda_{2,0}, D\right)$.

We observe that each of the sets

$$
\left\{t \omega: \omega \in \Xi_{1, l} \cup \Xi_{2, l}, t>0\right\}, \quad l \geqslant 1,
$$

contains just finitely many points $\bar{\lambda}_{k}$ such that $\left(\lambda_{k}, n_{k}\right) \in \Lambda_{2,0}$. This is why the embedding $\Xi\left(\Lambda_{2,0}\right) \subseteq S(0,1) \backslash$ int $J(D)$ holds.

Let $\Xi(\Lambda) \subseteq \operatorname{int} J(D)$. Then $\Lambda_{2,0}$ is a finite set. In this case $g_{2,0}$ is a polynomial. This is why $g_{2,0} \in W\left(\Lambda_{2}, 0, \mathbb{C}\right)$. We let $g_{1}=g_{1,0}+g_{2,0}, \Lambda_{1}=\Lambda_{1,0} \cup \Lambda_{2,0}, \Lambda_{2}=\emptyset$ and $g_{2}=0$.

Suppose now that $\Xi(\Lambda) \backslash$ int $J(D) \neq \emptyset$. In this case we let $\Lambda_{1}=\Lambda_{1,0}, \Lambda_{2}=\Lambda_{2,0}, g_{1}=g_{1,0}$ and $g_{2}=g_{2,0}$. The proof is complete.

\section{Fundamental PRINCIPLE}

In this concluding section we formulate and prove the main result of the work. First we provide some known results, which will be employed below.

Let $a, \varphi \in \mathbb{R}$. We consider a half-plane

$$
\Pi(a, \varphi)=\left\{z \in \mathbb{C}: \operatorname{Re}\left(z e^{-i \varphi}\right)<a\right\} .
$$

We let

$$
S_{\Lambda}(\varphi)=\min _{\left\{\lambda_{k(j)}\right\}} \lim _{\delta \rightarrow 0} \lim _{j \rightarrow \infty} \frac{\ln \left|q_{\Lambda}^{k(j)}\left(\lambda_{k(j)}, \delta\right)\right|}{\left|\lambda_{k(j)}\right|}, \quad q_{\Lambda}^{k}(z, \delta)=\prod_{\lambda_{\nu} \in B\left(\lambda_{k}, \delta\left|\lambda_{k}\right|\right), \nu \neq k}\left(\frac{z-\lambda_{\nu}}{3 \delta\left|\lambda_{\nu}\right|}\right)^{n_{\nu}},
$$

where the minimum is taken over all subsequences $\left\{\lambda_{k(j)}\right\}$ of the sequence $\left\{\lambda_{k}\right\}$ such that $\lambda_{k(j)} /\left|\lambda_{k(j)}\right| \rightarrow e^{-i \varphi}, j \rightarrow \infty$.

The sequence $\Lambda=\left\{\lambda_{k}, n_{k}\right\}$ is called almost real if $\Xi(\Lambda)=\{1\}$ and $\operatorname{Re} \lambda_{k}>0, k \geqslant 1$.

The following result was proved in Theorem 3.5 in work [11].

Theorem 4.1. Let $a \in \mathbb{R}, W$ be a closed non-trivial invariant subspace in $H(\Pi(a, 0))$ with an almost real spectrum $\Lambda=\left\{\lambda_{k}, n_{k}\right\}$. The following statements are equivalent.

1) Each function $g \in W$ is expanded into series (1.1) converging uniformly on compact sets in $\Pi(a, 0)$.

2) $S_{\Lambda}=0$.

We formulate also a result from work [9], see Corollary from Theorem 9.5, which resolves the fundamental principle problem for invariant subspaces of entire functions.

Theorem 4.2. Let $\Lambda=\left\{\lambda_{k}, n_{k}\right\}, \bar{n}(\Lambda)<+\infty$. The following statements are equivalent:

1) $S_{\Lambda}>-\infty$;

2) Each function $g \in W(\Lambda, \mathbb{C})$ is represented by series (1.1), which converges uniformly on compact sets in the plane.

We are going to prove an auxiliary result, which is of an independent interest.

Theorem 4.3. Let $\Lambda=\left\{\lambda_{k}, n_{k}\right\}, S_{\Lambda}=-\infty$. Then there exist numbers $\left\{d_{k, n}\right\}$ and numbers $k_{s}, 1=k_{1}<k_{2}<\ldots$, such that the series

$$
\sum_{s=1}^{\infty}\left(\sum_{k=k_{s}}^{k_{s+1}-1} \sum_{n=0}^{n_{k}-1} d_{k, n} z^{n} e^{\lambda_{k} z}\right)
$$

converges uniformly on compact sets in the plane and series 1.1) diverges at each point in the plane. 
Proof. According the assumptions and the definition of $S_{\Lambda}$, we find a subsequence of natural numbers $\{k(p)\}_{p=1}^{\infty}$ such that

$$
\frac{\ln \left|q_{\Lambda}^{k(p)}\left(\lambda_{k(p)}, \delta_{p}\right)\right|}{\left|\lambda_{k(p)}\right|} \leqslant-p
$$

where $(0,1 / 4) \ni \delta_{1} \geqslant \ldots \geqslant \delta_{p} \rightarrow 0$, and

$$
\left|\lambda_{k(p+1)}\right| \geqslant 2\left|\lambda_{k(p)}\right|, \quad p \geqslant 1 .
$$

We let

$$
\begin{aligned}
& \lambda_{k(p)}=r_{p} e^{i \varphi_{p}}, \quad B_{p}(c)=B\left(\lambda_{k(p)}, c \delta_{p} r_{p}\right), \\
& c_{p}=\sqrt{\left|q_{\Lambda}^{k(p)}\left(\lambda_{k(p)}, \delta_{p}\right)\right|}, \\
& g_{p}(z)=\frac{1}{2 \pi i} \int_{\partial B_{p}(5)} \frac{e^{\lambda z} d \lambda}{\left(\lambda-\lambda_{k(p)}\right) q_{\Lambda}^{k(p)}\left(\lambda, \delta_{p}\right)}, \quad p \geqslant 1 .
\end{aligned}
$$

We have:

Therefore,

$$
\left|q_{\Lambda}^{k(p)}\left(\lambda, \delta_{p}\right)\right| \geqslant 1, \quad \lambda \in \partial B_{p}(5)
$$

$$
\left|g_{p}(z)\right| \leqslant \sup _{\lambda \in \partial B_{p}(5)}\left|e^{\lambda z}\right| \leqslant \exp \left(\operatorname{Re}\left(\lambda_{k(p)} z\right)+5 \delta_{p} r_{p}|z|\right), \quad z \in \mathbb{C} .
$$

Let $K$ be an arbitrary compact set. By 4.6

$$
\left|g_{p}(z)\right| \leqslant e^{A r_{p}}, \quad z \in K, \quad p \geqslant 1,
$$

for some $A>0$. In view of 4.2 - (4.4) this implies:

$$
\sum_{p=p_{0}}^{\infty}\left|c_{p} g_{p}(z)\right| \leqslant \sum_{p=p_{0}}^{\infty} \exp \left(r_{p}(-p / 2+A)\right) \leqslant \sum_{p=p_{0}}^{\infty} e^{-r_{p}}<\infty, \quad z \in K .
$$

where $p_{0} \geqslant 2(A+1)$. Thus, the series

$$
g(z)=\sum_{p=1}^{\infty} c_{p} g_{p}(z)
$$

converges uniformly on each compact set in the plane. Employing the residues and the defintion of the function $q_{\Lambda}^{k(p)}\left(\lambda, \delta_{p}\right)$ and 4.4 for each $p \geqslant 1$, we obtain:

$$
c_{p} g_{p}(z)=d_{k(p), 0} e^{\lambda_{k(p)} z}+\sum_{\lambda_{k} \in B_{p}(1), k \neq k(p)} \sum_{n=0}^{n_{k}-1} d_{k, n} z^{n} e^{\lambda_{k} z}, \quad d_{k(p), 0}=\frac{c_{p}}{q_{\Lambda}^{k(p)}\left(\lambda, \delta_{p}\right)} .
$$

We also let $d_{k(p), n}=0, n=\overline{1, n_{k(p)}-1}$,

$$
d_{k, n}=0, \quad n=\overline{0, n_{k(p)}-1}, \quad \lambda_{k} \in B_{p}(1), \quad p \geqslant 1 .
$$

Since $\delta_{p} \in(0,1 / 4), p \geqslant 1$, by 4.3 there exist indices $k_{s}, 1=k_{1}<k_{2}<\ldots$, such that

$$
\sum_{s=1}^{m}\left(\sum_{k=k_{s}}^{k_{s+1}-1} \sum_{n=0}^{n_{k}-1} d_{k, n} z^{n} e^{\lambda \lambda_{k} z}\right)=\sum_{p=1}^{m} c_{p} g_{p}(z), \quad m \geqslant 1 .
$$

Thus, series 4.1 converges uniformly on compact sets in the plane.

By 4.4 and (4.2) we have:

$$
\left|d_{k(p), 0} e^{\lambda_{k(p)} z}\right|=\frac{1}{\sqrt{\left|q_{\Lambda}^{k(p)}\left(\lambda_{k(p)}, \delta_{p}\right)\right|}}\left|e^{\lambda_{k(p)} z}\right| \geqslant \exp \left((p / 2-|z|) r_{p}\right) \rightarrow \infty, \quad p \rightarrow \infty .
$$


Therefore, series 1.1 diverges at each point in the plane. The proof is complete.

Now we are in position to formulate and prove the main result of the work.

Theorem 4.4. Let $a, \varphi \in \mathbb{R}, W$ be a closed invariant subspace in $H(\Pi(a, \varphi))$ with the spectrum $\Lambda=\left\{\lambda_{k}, n_{k}\right\}, \bar{n}(\Lambda)<+\infty$. The following statements are equivalent.

1) Each function $g \in W$ is expanded into series (1.1) converging uniformly on compact sets in $\Pi(a, \varphi)$.

2) $S_{\Lambda}(\varphi)=0$ and $S_{\Lambda}>-\infty$.

Proof. By the assumptions, $\bar{n}(\Lambda)<+\infty$. Therefore, the system $\mathcal{E}(\Lambda)$ is incomplete in $H(\Pi(a, \varphi))$ and $W$ is a closed non-trivial invariant subspace in $H(\Pi(a, 0))$.

Assume that 1 ) holds. Suppose that $S_{\Lambda}=-\infty$. Then by Theorem 4.3 there exist numbers $\left\{d_{k, n}\right\}$ and indices $k_{s}, 1=k_{1}<k_{2}<\ldots$ such that series (4.1) converges uniformly on compact sets in the plane and series (1.1) diverges at each point in the plane. Let $g$ be the sum of series 4.1). Then $g \in W(\Lambda, \mathbb{C}) \subset W(\Lambda, \Pi(a, \varphi))$. It has been mentioned above that $W$ admits the spectral synthesis. This is why $W=W(\Lambda, \Pi(a, \varphi))$. According Statement 1$)$, the representation holds:

$$
g(z)=\sum_{k=1, n=0}^{\infty, n_{k}-1} a_{k, n} z^{n} e^{\lambda_{k} z}, \quad z \in \Pi(a, \varphi),
$$

and the series converges uniformly on compact sets in the half-plane $\Pi(a, \varphi)$. Then it follows from Lemma 2.2 that

$$
d_{k, n}=a_{k, n}, \quad n=\overline{0, n_{k}-1}, \quad k \geqslant 1 .
$$

This contradicts to the fact that series (1.1) diverges at each point in the plane. Thus, $S_{\Lambda}>$ $-\infty$.

Let $\left\{\lambda_{k(j)}\right\}$ be a subsequence $\left\{\lambda_{k}\right\}$ such that $\lambda_{k(j)} /\left|\lambda_{k(j)}\right| \rightarrow e^{-i \varphi}, j \rightarrow \infty$. We let $\Lambda_{0}=\left\{e^{i \varphi} \lambda_{k(j)}, n_{k(j)}\right\}$. Then $\Xi\left(\Lambda_{0}\right)=\{1\}$. This is why there exists an index $m$ such that $\Lambda_{0,0}=\left\{e^{i \varphi} \lambda_{k(j)}, n_{k(j)}\right\}_{j=m}^{\infty}$ is an almost real sequence. Let $\Lambda_{0,1}=\left\{\lambda_{k(j)}, n_{k(j)}\right\}_{j=m}^{\infty}$. According Statement 1), each function in the subspace $W\left(\Lambda_{0,1}, \Pi(a, \varphi)\right) \subset W(\Lambda, \Pi(a, \varphi))$ is expanded into series (1.1) converging uniformly on compact sets in $\Pi(a, \varphi)$. Therefore, each function in the subspace $W\left(\Lambda_{0,0}, \Pi(a, 0)\right)$ possesses the same property, but already on the compact sets in $\Pi(a, 0)$. Then by Theorem 4.1 the identity $S_{\Lambda_{0,0}}=0$ holds. This implies that $S_{\Lambda_{1}}=0$, where $\Lambda_{1}=\left\{\lambda_{k(j)}, n_{k(j)}\right\}$. Thus, $S_{\Lambda}(\varphi)=0$.

Assume now that Statement 2) holds and $g \in W(\Lambda, \Pi(a, \varphi))$. Since $\bar{n}(\Lambda)<+\infty$, the system $\mathcal{E}(\Lambda)$ is incomplete in $H(\Pi(a, \varphi))$. By Theorem 3.4, there exist sequences $\Lambda_{1}$ and $\Lambda_{2}$ such that $\Lambda=\Lambda_{1} \cup \Lambda_{2}, \Xi\left(\Lambda_{2}\right) \subset S(0,1) \backslash$ int $J(\Pi(a, \varphi))$ and the representation $g=g_{1}+g_{2}$ holds, where $g_{1} \in W\left(\Lambda_{1}, \mathbb{C}\right)$ and $g_{2} \in W\left(\Lambda_{2}, \Pi(a, \varphi)\right)$.

Since $S_{\Lambda}>-\infty$, then $S_{\Lambda_{1}}>-\infty$. Then by Theorem 4.1, the function $g_{1}$ is represented by series (1.1), which converges uniformly on compact sets in the plane.

The set $S(0,1) \backslash$ int $J(\Pi(a, \varphi))$ coincides with the point $e^{i \varphi}$. This is why $\Xi\left(\Lambda_{2}\right) \subseteq\left\{e^{i \varphi}\right\}$. Let $\Lambda_{2}=\left\{\lambda_{k(j)}, n_{k(j)}\right\}$. If $\Lambda_{2}$ is a finite sequence, then $g_{2}$ is an exponential polynomial and this completes the proof. Otherwise there exists an index $m$ such that $\Lambda_{2,0}=\left\{e^{i \varphi} \lambda_{k(j)}, n_{k(j)}\right\}_{j=m}^{\infty}$ is an almost real sequence. Let $\Lambda_{2,1}=\left\{e^{i \varphi} \lambda_{k(j)}, n_{k(j)}\right\}_{j=1}^{m-1}$ and $\Lambda_{2,2}=\left\{\lambda_{k(j)}, n_{k(j)}\right\}_{j=m}^{\infty}$. By Lemma 3.3 the representation $g_{2}=g_{2,1}+g_{2,2}$ holds, where $g_{2,1}$ is an exponential polynomial in the space $W\left(\Lambda_{2,1}, \mathbb{C}\right)$ and $g_{2,2} \in W\left(\Lambda_{2,2}, \Pi(a, \varphi)\right)$. Since $S_{\Lambda}(\varphi)=0$, then $S_{\Lambda_{2,0}}=0$. Then by Theorem 4.1, each function in $W\left(\Lambda_{2,0}, \Pi(a, 0)\right)$ is expanded into series (1.1) converging uniformly on compact sets in $\Pi(a, 0)$. Therefore, the function $g_{2,2}$ is expanded into series (1.1) converging uniformly on compact sets in $\Pi(a, \varphi)$. The proof is complete. 


\section{BIBLIOGRAPHY}

1. A.F. Leont'ev. Entire functions. Exponential series. Nauka, Moscow (1983). (in Russian).

2. I.F. Krasičkov-Ternovskiĭ. Invariant subspaces of analytic functions. I. Spectral analysis on convex regions // Matem. Sborn. 87(129):4, 459-489 (1972). [Math. USSR-Sb. 16:4, 471-500 (1972).]

3. I.F. Krasičkov-Ternovski1. Invariant subspaces of analytic functions. II. Spectral synthesis of convex domains // Matem. Sborn. 88(130):3(7), 3-30 (1972). [Math. USSR-Sb. 17:1, 1-29 (1972).]

4. A.A. Gol'dberg, B.Ya. Levin, I.V. Ostrovskii. Entire and meromorphic functions // Itogi Nauki i Tekhniki. Ser. Sovrem. Probl. Mat. Fund. Napr. 85,5-185 (1991). (in Russian).

5. A.S. Krivosheev. A fundamental principle for invariant subspaces in convex domains // Izv. RAN. Ser. Matem. 68:2, 71-136 (2004). [Izv. Math. 68:2, 291-353 (2004).]

6. O.A. Krivosheeva, A.S. Krivosheev. A criterion for the fundamental principle to hold for invariant subspaces on bounded convex domains in the complex plane // Funkts. Anal. Pril. 46:4, 14-30 (2012). [Funct. Anal. Appl. 46:4, 249-261 (2012).]

7. A.S. Krivosheev, O.A. Krivosheeva. A basis in an invariant subspace of analytic functions // Matem. Sborn. 204:12, 49-104 (2013). [Sb. Math. 204:12, 1745-1796 (2013).]

8. A.S. Krivosheev, O.A. Krivosheeva. Fundamental principle and a basis in invariant subspaces // Matem. Zamet. 99:5, 684-697 (2016). [Math. Notes. 99:5, 685-696 (2016).]

9. A.S. Krivosheev, O.A. Krivosheyeva. A basis in invariant subspace of entire functions // Alg. Anal. 27:2, 132-195 (2015). [St. Petersburg Math. J. 27:2, 273-316 (2016).]

10. A.S. Krivosheyev, O.A. Krivosheyeva. A closedness of set of Dirichlet series sums // Ufimskij Matem. Zhurn. 5:3, 96-120 (2013). [Ufa Math. J. 5:3, 94-117 (2013).]

11. O.A. Krivosheyeva, A.S. Krivosheyev. A representation of functions from an invariant subspace with almost real spectrum // Alg. Anal. 29:4, 82-139 (2017). [St. Petersburg Math. J. 29:4, 603-641 (2018).]

12. A.F. Leont'ev. Sequences of exponential polynomials. Nauka, Moscow (1980). (in Russian).

13. B.Ya. Levin. Distribution of zeros of entire functions. Gostekhizdat, Moscow (1956). [Amer. Math. Soc., Providence, RI (1980).]

14. B.N. Khabibullin. On the growth of entire functions of exponential type along the imaginary axis // Matem. Sborn. 180:5, 706-719 (1989). [Math. USSR-Sb. 67:1, 149-163 (1990).]

15. P. Malliaven, L. Rubel. On small entire functions of exponential type with given zeros // Bull. Soc. Math. France 89, 175-201 (1961).

Alexander Sergeevich Krivosheev,

Institute of Mathematics,

Ufa Federal Research Center, RAS,

Chernyshevsky str. 112,

450008, Ufa, Russia

Olesya Aleksandrovna Krivosheeva,

Bashkir State University,

Zaki Validi str. 32,

450076, Ufa, Russia

E-mail: kriolesya2006@yandex.ru 